
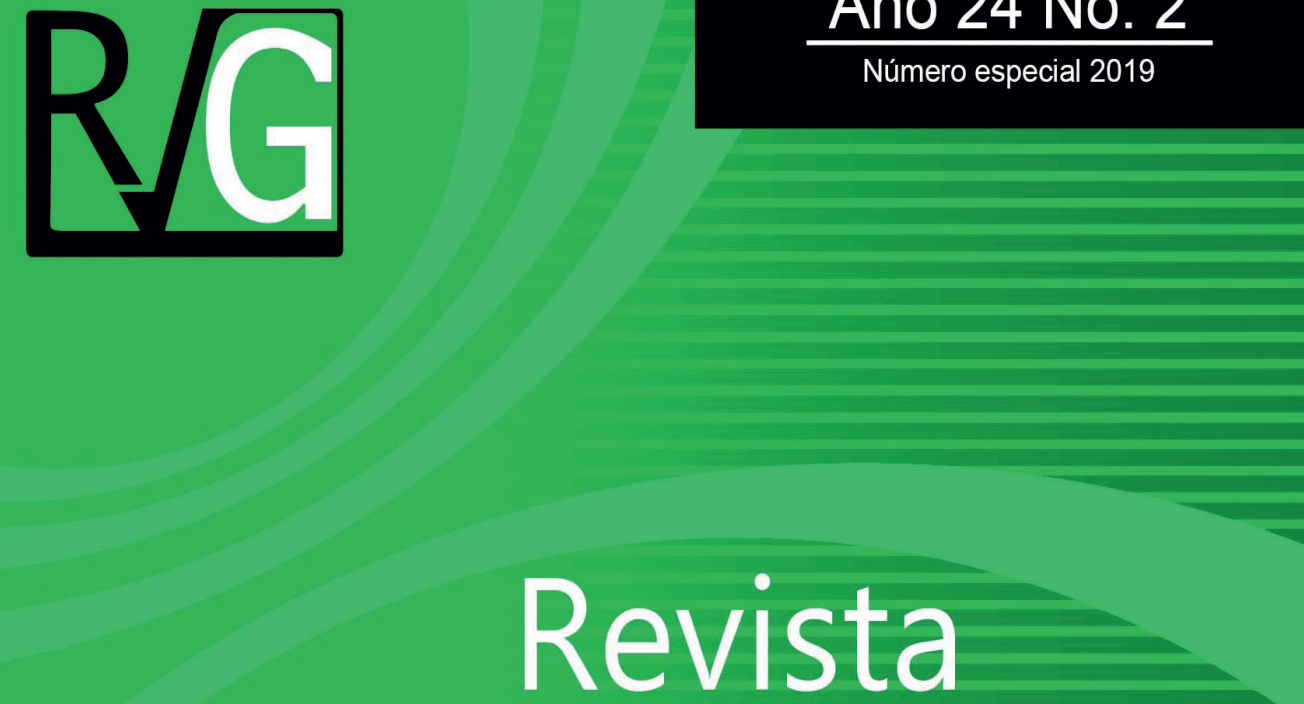

Venezolana de

$\frac{1}{6}$

$\frac{\mathbb{O}}{\frac{1}{0}}$
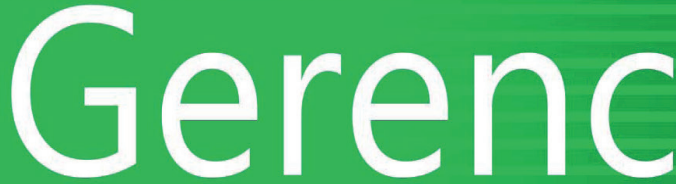

0

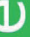

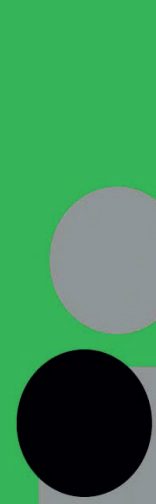

ro

u

$>$

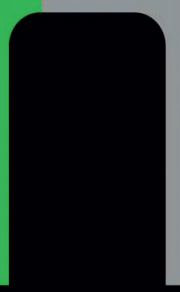




\title{
Iniciativas de Investigación, Ciencia, Tecnología e Innovación: Centro de Innovación y Desarrollo Empresarial del Cauca (CIDECAUCA), Institución Universitaria Colegio Mayor del Cauca ${ }^{1}$
}

\author{
Didier Rubén, Cordoba Lozada²
}

\section{Estrategia de apropiación}

Debido a la poca conexión entre las misiones de docencia, investigación y relacionamiento con el entorno, y a la falta de procesos de innovación para que las empresas generen condiciones de desarrollo y de competitividad en el territorio caucano, se ha generado una deficiencia en la articulación UniversidadEmpresa - Estado y Sociedad para la generación de capacidades de gestión y desarrollo territorial. Esto ha desplegado grandes impactos negativos en el desarrollo económico de la sociedad, ya que no ha permitido que los pequeños empresarios generen capacidades que impulsen el desarrollo del sector productivo en el Departamento del Cauca.

Como una estrategia de posicionamiento con respecto a la dinámica universidad - empresa - estado, y dando respuesta a la

\footnotetext{
Recibido: 30-09-19 Aceptado: 29-10-19
}

Conferencia presentada en el marco del III Encuentro Internacional de Investigadores y Estudiantes de la Red Académica Internacional de Estudios Organizacionales en América Latina, el Caribe e Iberoamérica realizado del 1 al 7 de Diciembre de 2019, Popayán, Colombia

2 Director del Centro de Innovación y Desarrollo Empresarial del Cauca (Cidecauca) Institución Universitaria Colegio Mayor del Cauca, Docente Investigador del Grupo de Investigación en Administración, Finanzas y Economía (GIFIN), Institución Universitaria Colegio Mayor del Cauca, Ingeniero de Minas Fundación Universitaria de Popayán - Magister en Administración de Empresas con Énfasis en Dirección de Proyectos Universidad Viña del Mar - Chile, Especialista Sistemas Gerenciales de Ingeniería - Gerencia de Proyectos - Pontificia Universidad Javeriana. ORDCID: 0000-0003-2707-7772 
necesidad identificada y plasmada en el Plan de Desarrollo de la Institución Universitaria Colegio Mayor del Cauca vigencia 2016-2020, articulado al eje estratégico Relacionamiento Externo, quien propone que se participe activamente con los diferentes sectores de la academia mediante alianzas interinstitucionales como lo expresa el Ministerio de Educación Nacional (2013): Las relaciones de las IES con el Estado, la empresa y la sociedad civil $y$ las relaciones entre las instituciones encargadas de la educación deben fortalecerse creando espacios de diálogo $y$ de construcción conjunta.

En este contexto de creación de espacios el Grupo de Investigación en Administración, Finanzas y Economía (GIFIN) de la Institución Universitaria Colegio Mayor del Cauca en el ejercicio de participación colaborativa en el aporte acciones al Plan de Desarrollo 2016-2020 "Gestión Transformadora con calidad Humana" identificó que el mecanismo de respuesta al Eje Estratégico 1 : Fortalecimiento Académico - Investigativo, iniciativas que generen posicionamiento de la Institución a nivel regional, nacional e internacional mediante la articulación de la UNIVERSIDAD-EMPRESA-ESTADOSOCIEDAD fuese por medio de una dinámica que permitiese a la Institución en materia del relacionamiento con el sector externo el fortalecimiento de la Institución con los actores del tejido empresarial del departamento del Cauca el resultado del ejercicio es la creación mediante Resolución Institucional 008 de 2018 del Centro de Innovación y Desarrollo Empresarial del Cauca (CIDECAUCA).

EICentro de Innovación y Desarrollo Empresarial del Cauca - CIDECAUCA $\circledR$ - es una dinámica de la Institución
Universitaria Colegio Mayor del Cauca para fortalecer el relacionamiento con el sector productivo, organizaciones públicas y privadas, ofertando servicios en asesorías, asistencias, consultorías, estudios de mercado, gestiones administrativas, gestión de proyectos sociales o productivos entre otros, que les permitirán a dichas organizaciones, negocios, proyectos de emprendimiento y empresas comerciales $\mathrm{o}$ industriales posicionarse en la región y cumplir con sus objetivos.

EI CIDECAUCA ${ }^{\circledR}$, es una marca registrada, que propende identificar necesidades de un entorno empresarial basado en la micro y la mediana empresa de la región, articularla a soluciones que el medio académico puede ofertar con el propósito de coadyuvar al cierre de la brecha entre la universidad el estado y la sociedad.

\section{Apropiación social de conocimiento.}

El gran salto a la denominada 'sociedad del conocimiento' implica, en principio, de parte de las universidades y los gobiernos, la implementación de mecanismos de participación y socialización de la ciencia y la tecnología, esencialmente. De ahí que empiecen a aparecer políticas públicas orientadas a la divulgación, que deben atender los posibles usos de los conocimientos científicos y tecnológicos de los que la sociedad podría sacar provecho, lo cual quiere decir que representan los "diversos intereses sociales y políticos, que se corresponden, a su vez, con determinadas preferencias ideológicas, ideales sobre la ciencia, y con distintos tipos de actuación y formas de relacionarse con el resto de las culturas" (Sanz, 2008, pp. 87-88). Ello implica la 
participación social igualitaria, más y mejores canales de comunicación que permitan entablar una conversación concertada, así como la formación de opinión pública, es decir, llegar a un verdadero "contrato social" para la ciencia y la tecnología que asocie a todos los actores de la sociedad (Lozano, 2008. En Colombia, por ejemplo, se ha comenzado a discutir alrededor de la definición e inclusión de políticas públicas en los planes de gobierno municipal, departamental y nacional; resultado de esto es la Ley de Ciencia y Tecnología y la inclusión de Colciencias como departamento administrativo. Sin embargo, estas iniciativas se han dado de manera fragmentada, lo que ha impedido obtener resultados directos en la sociedad colombiana. (Lozano, 2005). Apuntar, entonces, a una gobernanza política en ciencia y tecnología, que se refleje en mejores programas, planes y proyectos científicos y tecnológicos que contribuyan al mejoramiento de la calidad de vida de los ciudadanos, implica necesariamente que quienes viven dentro de una misma comunidad ayuden a definir sus necesidades más prioritarias

Aunque el país ha realizado un esfuerzo importante en el impulso hacia la apropiación de la Ciencia, la Tecnología y la Innovación, estos esfuerzos no han sido suficientes, como lo señala el documento Informe Nacional de la Estrategia de Apropiación de la Ciencia, la Tecnología e Innovación: "las actividades que concretamente le pusieron el acento al fortalecimiento de la Apropiación Social del Conocimiento entre los años 2005 y 2009, en su mayoría éstas se basan en modelos deficitarios de comunicación, y están orientadas a un público de clase media, urbano, con acceso a educación formal.
Estas actividades se enfocaron en la transmisión de conocimientos científico tecnológicos, con el fin de aumentar una valoración positiva hacia la ciencia y la tecnología. Son pocas las acciones sustentadas en modelos democráticos que le permitan a unos "otros" re-conocer su papel fundamental en la construcción de conocimiento" (Documento: Estrategia de Apropiación de la Ciencia, la Tecnología e Innovación, 2012).

La apropiación social del conocimiento se entiende como un proceso que implica, por un lado, la disposición de los conocimientos científicos y tecnológicos en un escenario y lenguaje comunes para la sociedad; y por otro, que el ser humano hizo suyos tales conocimientos como elementos útiles y necesarios para su beneficio y provecho. Apropiación social del conocimiento, desde la óptica de la sociedad del conocimiento, significa, entonces, la democratización del acceso y uso del conocimiento científico y tecnológico, como estrategia para su adecuada transmisión y aprovechamiento entre los distintos actores sociales, que derivará en el mejoramiento de la calidad de vida de las comunidades y sus integrantes. Esta afirmación implica que las universidades (como el lugar donde se alberga la mayor parte de las comunidades científicas), en armonía con los gobiernos, deberán prestar atención a la tarea de llevar a públicos amplios, más allá de los círculos académicos, el conocimiento que la actividad científica y tecnológica producen y que podría beneficiarlos. Por eso, el establecimiento de focos de intervención universitaria para la solución de problemas sociales y la producción de nuevo conocimiento significa un reto epistémico en cuanto al modo dominante de producción de conocimientos en 
las universidades, porque estos no deben quedar reducidos a las elites intelectuales. Así, apropiar, también entra en juego con lo ideológico, en la medida que será necesario abandonar la idea de la extensión universitaria como una cuestión de asistencia, para llegar a la democratización del conocimiento como un compromiso social de construcción conjunta, lo que acarrea la responsabilidad de una dedicación especial respecto a los modos como se comunican y entregan los saberes a la comunidad (Carrizo, 2001).

\section{Estrategia de apropiación social para CIDECAUCA.}

Así pues, debe asumirse como concepto de apropiación social la construcción sociocultural fruto del proceso de comprensión e intervención en las relaciones sociales de objetos innovadores desde la lógica socio técnica más amplia, construida a partir de la participación de los diversos grupos sociales que generan conocimiento desde su quehacer cotidiano, sea profesional o sea como ser humano concreto.

Por lo anterior, el componente de apropiación social de conocimiento para esta propuesta responde a la necesidad de generar mecanismos que propicien la inclusión del empresario y la comunidad en general a la información y el conocimiento de los temas abordados en el proyecto. De esta forma se plantea alrededor de las cuatro líneas de acción planteadas en la Estrategia Nacional de apropiación de CTel de Colciencias.

\section{Figura 1}

\section{Líneas de desarrollo de la Estrategia Nacional de CTel}

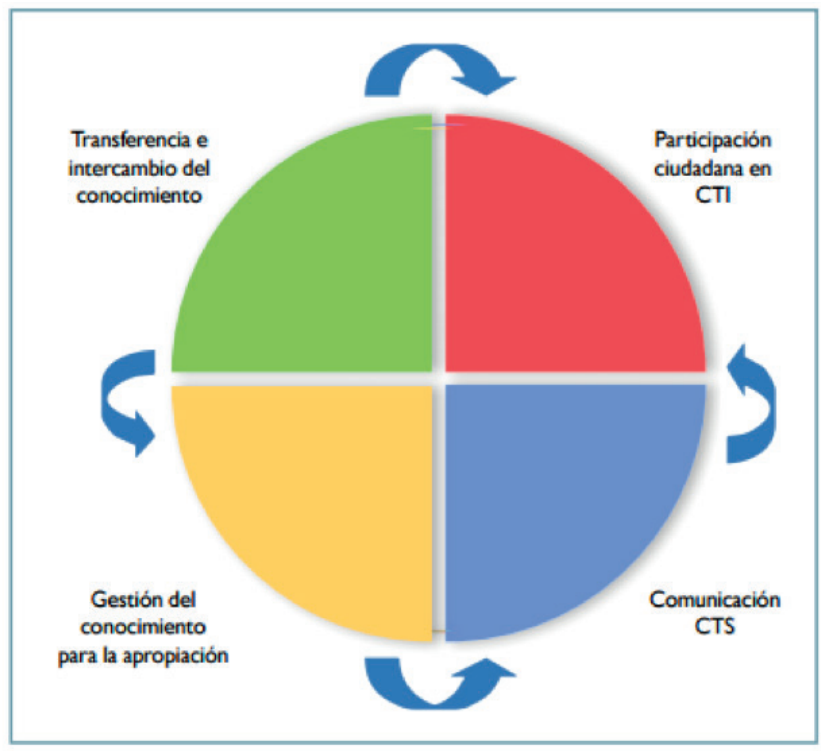

Fuente: Colciencias (2019) 


\section{Actividades de apropiación}

\section{a. Intercambio y transferencia} de conocimiento: "Busca promover procesos de generación y uso del conocimiento más democráticos, responsables y respetuosos de las especificidades culturales y sociales de las comunidades "objeto" o "beneficiarias" de proyectos de investigación o innovación”. (Estrategia Nacional de ASCTI)

- Eventos para socializar los resultados de los proyectos

- Giras de oportunidades (Taller de experiencias, dirigido a jóvenes para acercarlos a vivencias y actividades concretas en relación con el mundo del emprendimiento.

- Laboratorio de la Innovación (identificación de ideas de emprendimiento y acompañamiento, aceleración e innovación de esos emprendimientos y que se desea refinar para lograr su desarrollo)

- Congreso, Foros

- Sistematización de experiencias

b. Comunicación en Ciencia, Tecnología y sociedad: "tiene por objetivo favorecer el desarrollo de proyectos de Comunicación sobre las relaciones Ciencia, Tecnología y Sociedad. Más que la simple unión de esos tres conceptos en una sigla, se trata de una perspectiva que busca resaltar las interacciones entre ellos, y desde ahí, convertirse en una propuesta concreta de trabajo, que hace el Programa de ASCTI para desarrollar con los diversos actores que gestionan proyectos en esta área." (Estrategia Nacional de ASCTI).

- Formación de colectivos de comunicación con jóvenes interesados en el tema emprendimiento

- Portal web informativo con información del proyecto

- Dinamización de redes sociales

c. Gestión del conocimiento para la apropiación: "implica no solo la formación de recursos humanos, sino también desarrollar mecanismos de comprensión e interpretación de las relaciones tecnociencia y sociedad. Esto significa generar metodologías que permitan leer las diversas formas de ser apropiado el conocimiento científico y técnico en la sociedad colombiana, por los diversos grupos e individuos que la componen" (Estrategia Nacional de ASCTI).

- Indicadores de apropiación

- Centro de documentación de buenas prácticas y lecciones aprendidas de experiencias de innovación en el Cauca

- Comunidades de aprendizaje como eje de soporte a la plataforma.

- Esta obra está bajo una licencia de Creative Commons Reconocimiento-NoComercialCompartirlgual 3.0 Unported. http://creativecommons.org/licenses/by-nc-sa/3.0/deed.es_ES 